\title{
Plasma fatty acid-binding protein 4 (FABP4) level is associated with abnormal QTC interval in patients with stable angina and chronic kidney disease
}

Chao-Ping Wang ${ }^{1,6}$, Chia-Chang Hsu², Wei-Chin Hung ${ }^{1}$, Teng-Hung Yu ${ }^{1}$, Cheng-Ching Wu ${ }^{1}$, I-Ting Tsai ${ }^{4}$, Wei-Hua Tang ${ }^{5}$, Fu-Mei Chung ${ }^{1}$, Jer-Yiing Houng ${ }^{7}$, Yau-Jiunn Lee ${ }^{8}$ and Yung-Chuan Lu ${ }^{3,6^{*}}$ (D)

\begin{abstract}
Background: Fatty acid-binding protein 4 (FABP4) (also known as adipocyte FABP or adipocyte P2) is expressed in adipocytes, macrophages, and capillary endothelial cells. Previous studies have shown associations among plasma FABP4, insulin resistance, metabolic syndrome, diabetes mellitus, greater coronary plaque burden, coronary artery disease, heart failure, and mortality. However, little is known about the relationship between FABP4 level and prolonged QT interval. The aim of this study was to investigate whether plasma FABP4 level is associated with a prolonged QT interval by analyzing 12-lead electrocardiograms (ECGs) in patients with stable angina and chronic kidney disease (CKD).

Methods: This study included 397 consecutive patients with stable angina and CKD who were enrolled in a disease management program. Plasma FABP4 concentrations were measured using enzyme-linked immunosorbent assays. A 12-lead ECG recording was obtained from each patient. We assessed the relationships between FABP4 levels (both as a continuous variable and stratified by tertile) at admission and corrected QT (QTc) prolongation.

Results: Patients with an abnormal QTc interval had higher median plasma FABP4 levels than those with borderline and normal QTc intervals (15.9 ng/mL vs. $10.2 \mathrm{ng} / \mathrm{mL}$ vs. $8.5 \mathrm{ng} / \mathrm{mL}$, respectively, $P<0.0001)$. Statistically significant associations were observed between plasma FABP4 levels and QTc interval $(\beta=0.267, P<0.0001)$. Using multivariate and trend analyses, a higher concentration of plasma FABP4 level was independently associated with QTc prolongation in patients with stable angina and CKD.

Conclusion: In this study, plasma FABP4 levels were significantly higher in the patients with an abnormal QTc interval and were correlated with QTc prolongation. Further studies are required to elucidate whether plasma FABP4 plays a role in the pathogenesis of QTc prolongation.
\end{abstract}

Keywords: Fatty acid-binding protein 4, Stable angina, Chronic kidney disease, QTc interval

\footnotetext{
* Correspondence: gregory.yclu@msa.hinet.net

${ }^{3}$ Division of Endocrinology and Metabolism, Department of Internal

Medicine, E-Da Hospital, I-Shou University, No. 1, Yi-Da Rd, Jiau-Shu Village,

Yan-Chao District, Kaohsiung 82445, Taiwan

${ }^{6}$ School of Medicine for International Students, College of Medicine, I-Shou

University, Kaohsiung 82445, Taiwan

Full list of author information is available at the end of the article
}

(c) The Author(s). 2019 Open Access This article is distributed under the terms of the Creative Commons Attribution 4.0 International License (http://creativecommons.org/licenses/by/4.0/), which permits unrestricted use, distribution, and reproduction in any medium, provided you give appropriate credit to the original author(s) and the source, provide a link to the Creative Commons license, and indicate if changes were made. The Creative Commons Public Domain Dedication waiver (http://creativecommons.org/publicdomain/zero/1.0/) applies to the data made available in this article, unless otherwise stated. 


\section{Background}

The QT interval on surface electrocardiograms (ECGs) represents the time from onset of ventricular depolarization to completion of repolarization, and several studies of older adults and patients with myocardial ischemia or infarctions have reported an association between prolongation of this interval and ventricular arrhythmias that could trigger ventricular fibrillation and sudden cardiac death [1-3]. Furthermore, prolonged heart rate-corrected QT (QTc) interval has also been associated with an increased risk of coronary heart disease and cardiovascular disease mortality in the general population $[4,5]$. A prolonged QTc interval is a complex trait which can be affected by both genetic and environmental factors (e.g. age and sex) $[6,7]$. In addition, QTc prolongation is very common and is associated with several risk factors in patients with chronic kidney disease (CKD) [8]. CKD is associated with elevated inflammatory markers and increased with progression of renal failure [9]. A previous study suggested that elevated inflammatory mediators and activation of the renin-angiotensin system contribute to arterial calcification and vascular atherosclerosis through enhanced production of reactive oxygen species in patients with CKD [10]. Previous studies have also demonstrated that during reperfusion of ischemic myocardium, cytokines such as platelet-activating factor generated by activated neutrophils can cause some arrhythmias $[11,12]$. In addition, Chung et al. showed that elevated C-reactive protein (CRP) may reflect an inflammatory state that promotes the persistence of atrial fibrillation [13]. Hence, increased levels of inflammatory mediators in patients with CKD may provoke cardiac fibrosis, vascular damage, sympathetic overactivity, and ion channel malfunction [9-14], resulting in a higher risk of cardiac arrhythmias and death.

Fatty acid-binding protein 4 (FABP4) is an adipokine produced by adipose tissues. It is a carrier protein which has been shown to carry lipophilic compounds including fatty acids between intra- and extra-cellular membranes $[15,16]$. In addition, it has been shown to affect inflammation, insulin resistance, thrombogenicity, and other metabolic pathways [17-19]. A previous study suggested that FABP4 may have a negative inotropic effect on cardiomyocytes [20]. Furthermore, the expression of FABP4 in adipocytes has been positively associated with mortality [21], incident diabetes mellitus [22], greater coronary plaque burden [23], coronary artery disease (CAD) [24], and heart failure (HF) [25]. Moreover, FABP4 plasma concentrations have been reported to potentially be an early clinical marker of renal function derangement in patients with type 2 diabetes [26]. Furthermore, in isolated rat cardiomyocytes, FABP4 was shown to acutely depress shortening amplitude and intracellular systolic peak $\mathrm{Ca}(2+)$ in a dose-response fashion [20]. This suggests that FABP4 may play an important role in cardiac depolarization and possibly cardiac arrhythmias. Given the association between QT interval prolongation, CKD, and inflammation, and given the inflammatory mediator effects of FABP4 and its relationship to cardiovascular disease and cardiac arrhythmias, we hypothesized that FABP4 may be independently associated with QTc interval prolongation in humans.

In addition, plasma FABP4 level has been positively associated with coronary plaque burden, CAD, HF, and mortality [21, 23-25]. These observations suggest that FABP4 may play a role in the development of cardiovascular damage, and it is reasonable to assume that plasma FABP4 may be associated with conductive system injury. However, to the best of our knowledge, little is known about the relationship between FABP4 level and cardiac electro-pathology. Therefore, the aim of this study was to investigate whether FABP4 levels were associated with a prolonged QTc interval in a cohort of patients with stable angina and CKD.

\section{Methods \\ Study population}

From June 2006 to June 2015, 397 consecutive consenting patients with a clinical diagnosis of stable angina underwent angiography for the first time at the Cardiovascular Clinic of E-Da Hospital. The estimated glomerular filtration rate (eGFR) was calculated for each patient using the CKD-EPI two-level race equation within 3 to 6 months of admission [27]. Patients with CKD stage II-IV were eligible for inclusion into this study, and their baseline clinical characteristics were recorded. Stable angina pectoris was defined as retrosternal chest discomfort, precipitation by exertion, prompt relief within $30 \mathrm{~s}$ to $10 \mathrm{~min}$ with rest or nitroglycerine, and chest pain related to effort with no evidence of recent deterioration or rest pain in the past 6 months. The exclusion criteria were patients with inflammatory diseases (including sepsis or infection), collagen diseases, liver diseases, malignancy, steroid use, a bundle branch block pattern, and a history of psychosis. In addition, patients taking medications which may have influenced the QT interval including psychotropic medications and class I (e.g. flecainide, quinidine, mexiletine, and procainamide) and class III (e.g. dronedarone, amiodarone, and vernakalant) anti-arrhythmic medications were also excluded from the study. Written informed consent was obtained from each patients before entry into this study, which was approved by the Human Research Ethics Committee at our institution.

We used online software (https://www.anzmtg.org/ stats/PowerCalculator/PowerANOVA) to calculate the required sample size. When the number of groups was set at three, the minimum acceptable power level was 
0.80. Furthermore, the effect size was set at a medium effect size (0.25) and the significance level was set at 0.05 . The results showed a sample size of 52.4 per group. The formula used to calculate the effect size was as follows:

$$
\eta^{2}=\frac{\text { Sum of squares }}{\text { Total sum of squares }}
$$

Before the coronary angiography examination, the detailed records of each patient with regards to medical and personal history were reviewed. The following cardiovascular disease risk factors were assessed. Smoking status was classified as non-smoker, former smoker (having stopped smoking for $\geq 1$ year), or current smoker. Current and former smokers were grouped for analysis and compared to the never smokers. Patients with a current or prior diagnosis of type 2 diabetes and those receiving medical therapy for type 2 diabetes were defined as having type 2 diabetes in accordance with World Health Organization guidelines [28]. Hypertension was defined as persistent elevation of systolic blood pressure (SBP) $(\geq 140 \mathrm{mmHg})$ and/or diastolic blood pressure (DBP) $(\geq 90 \mathrm{mmHg})$. Patients who were receiving antihypertensive therapy were also defined as having hypertension. The following were used to define hyperlipidemia according to the Adult Treatment Panel III criteria [29]: high-density lipoprotein cholesterol (HDL-C) levels of $<35 \mathrm{mg} / \mathrm{dL}$ and $<39$ $\mathrm{mg} / \mathrm{dL}$ for men and women, respectively; low-density lipoprotein cholesterol (LDL-C) level of $\geq 130 \mathrm{mg} / \mathrm{dL}$; total cholesterol level of $\geq 200 \mathrm{mg} / \mathrm{dL}$; triglyceride (TG) level of $\geq 150 \mathrm{mg} / \mathrm{dL}$; or currently receiving antidyslipidemic medications.

\section{Laboratory measurements}

Before the coronary angiography examination, plasma biochemical parameters were measured in all of the patients after fasting for $8 \mathrm{~h}$. All biochemical analyses were performed at the E-Da Hospital laboratory within $2 \mathrm{~h}$ of the blood samples being drawn. A parallel, multichannel analyzer (Hitachi 7170A, Hitachi Ltd., Tokyo, Japan) was used to measure complete blood cell count, lipid profile (including plasma TGs, total cholesterol, LDL-C, and HDL-C), and levels of uric acid, albumin, glucose, sodium, calcium, potassium and serum creatinine. In addition, the concentrations of plasma FABP4 concentration was determined using an enzyme-linked immunosorbent assay kits (R\&D Systems Inc., Minneapolis, MN, USA). The dilution and standard curves were parallel, and the intra- and inter-assay coefficients of variation of the assay were 3.4 to $5.8 \%(n=3)$ and 3.1 to $6.2 \%(n=4)$, respectively. A high-sensitivity method was used to measure levels of plasma CRP with an IMMAGE system (Beckman Coulter, Immunochemistry Systems, Brea, CA, USA) that had a detection limit of $0.2 \mathrm{mg} / \mathrm{L}$. The intra-assay coefficient of variation was 4.2 to $8.7 \%$ for highsensitivity (hs)-CRP. Samples were assessed in duplicate in a single experiment.

\section{Angiographic definitions}

Coronary angiography was performed following standard techniques, and the degree of stenosis was assessed using quantitative coronary angiography (QCA). A minimum of two experienced interventional cardiologists who were blinded to the clinical information and serologic parameters of the patients evaluated the angiographic data. Two scoring systems were used to classify the results. The first referred to the total number of diseased vessels, in which 1-, 2-, or 3-vessel disease was defined as a $>75 \%$ reduction in the internal diameter, and a stenotic diameter of the left main coronary artery not exceeding 50\%. The second scoring system was the modified Gensini scoring system, in which each coronary segment is scored depending on the importance and size of the vessel, with scores ranging from 0.5 to 5.0. For example, $0-25 \%$ stenosis of the coronary artery lumen is scored $2,26-50 \%$ is scored $4,51-75 \%$ is scored $8,76-90 \%$ is scored $16,91-99 \%$ is scored 32, and $100 \%$ is scored 64 . The modified Gensini index is then calculated as the sum of the total scores for each segment [30,31].

\section{Electrocardiogram, QT and QTc interval measurements}

A minimum of two cardiologists who were blinded to this study manually measured the QT intervals. Twelvelead ECGs were recorded using a standardized protocol upon enrollment into the study. The QTc of lead II was measured and analyzed in this study. QT interval was defined as the interval between the first deflection of the QRS complex and the end of the $T$ wave, which was measured using a tangent extending from the steepest section of the $\mathrm{T}$ wave downslope to where it crossed the T-P segment. All QT and RR intervals were averaged over three consecutive complexes in sinus rhythm and over all complexes on the 10-s lead II rhythm strip on the 12-lead ECGs in other rhythms.

The QTc intervals were calculated using Bazett's formula $(\mathrm{QTC}=\mathrm{QT} / \sqrt{\mathrm{RR}})[32,33]$. The ECG tracings were blinded and analyzed by two independent coders. Interreader discrepancies were resolved by direct comparison and adjudicated by another supervisor. A coefficient of reliability of 0.995 and Pearson's correlation coefficient of 0.995 were calculated in the inter-reader reproducibility assessment for QT measurements. Comparisons of inter-reader QT measurements using the paired $t$-test did not reach statistical significance $(P=0.35)$. Extremely 
rapid (> $150 \mathrm{bpm})$ and extremely slow $(<40 \mathrm{bpm})$ heart rate recordings were excluded [34, 35]. In this study, QTc prolongation was categorized into three sexspecific categories as reported in a previous study [36], with cutoff values of $\leq 450 \mathrm{~ms}$ (normal), 451 to $470 \mathrm{~ms}$ (borderline), and $>470 \mathrm{~ms}$ (prolonged) for women, and $\leq 430 \mathrm{~ms}$ (normal), 431 to $450 \mathrm{~ms}$ (borderline), and $>450 \mathrm{~ms}$ (prolonged) for men.

\section{Statistical analysis}

Data are expressed as mean \pm SD or median and interquartile range (IQR). We used the Kolmogorov-Smirnov test to evaluate the normality of variables. Normally distributed variables were compared using one-way analysis of variance (ANOVA) with Tukey's pairwise comparison. Before performing the statistical tests, we logarithmically transformed levels of serum TGs, creatinine, FABP4, and hs-CRP to achieve a normal distribution. Categorical variables are presented as frequency and/or percentages, and the $x^{2}$ test was used for inter-group comparisons. Multivariate logistic regression analysis using the existence of an abnormal QTc interval as a dependent variable was conducted to determine the relative contributions to the outcome variable made by each variable.

We also recorded the occurrence of major adverse cardiac events (MACEs) after the patients had been discharged from the hospital, defined as all-cause mortality or re-hospitalization for a repeat percutaneous coronary intervention (PCI) or coronary artery bypass grafting, or for a cardiovascular-related illness including HF, recurrent angina pectoris, and nonfatal reinfarction. We also divided the distribution of plasma FABP4 levels into tertiles as follows: first tertile, $\leq 7.4 \mathrm{ng} / \mathrm{mL}$; second tertile, 7.5 to $16.25 \mathrm{ng} / \mathrm{mL}$; and third tertile, $>16.25 \mathrm{ng} / \mathrm{mL}$. Laboratory features and ECG data in each tertile were described and tested for trend across plasma FABP4 tertiles using linear regression analysis and the Cochran-Armitage trend test for categorical variables including MACEs, medications, and other diseases. Correlations among FABP4 and biochemical and anthropometric parameters were analyzed using Pearson's correlation analysis, and associations among QTc interval and FABP4 level and eGFR were analyzed using linear regression analysis. All tests were two-tailed, and a $P$-value of $<0.05$ was considered to be statistically significant. Statistical analysis was performed using JMP software version 7.0 for Windows (SAS Institute, Cary, NC, USA).

\section{Results}

Table 1 presents the baseline clinical, angiographic and biochemical data of the participants according to QTc prolongation status. One hundred and 71patients were defined as having a normal QTc interval, 88 patients were defined as having borderline QTc prolongation, and 138 patients were defined as having an abnormal QTc interval. The abnormal QTc interval group had a significantly higher serum FABP4 level than the borderline and normal QTc interval groups $(15.9 \mathrm{ng} / \mathrm{mL}$ [IQR 6.7 to 41.4 ] vs. $10.2 \mathrm{ng} / \mathrm{mL}$ [IQR 5.3 to 19.7 ] vs. $8.5 \mathrm{ng} / \mathrm{mL}$ [IQR 5.2 to 14.4], respectively, $P<0.0001)$. In addition, the abnormal QTc interval group were older $(P=0.003)$ and had higher prevalence rates of diabetes mellitus $(P=0.003)$, hypertension $(P=0.0002)$, and HF $(P<0.0001)$. Furthermore, the abnormal QTc interval group had a higher white blood cell count, higher levels of fasting glucose, HbA1c, creatinine, uric acid, and blood urea nitrogen, a lower prevalence rate of hyperlipidemia, and lower levels of sodium, albumin, hematocrit, and hemoglobin than the normal QTc interval group. Moreover, the abnormal QTc interval group (prolonged QT interval) had a higher hs-CRP level and a lower eGFR than the normal and borderline QTC interval groups. There were no significant differences in smoking status, body mass index (BMI), waist circumference, smoking status, SBP, DBP, levels of calcium, potassium, TGs, HDL-C, and LDL-C, total cholesterol, number of diseased coronary arteries, Gensini score, PCIs, number of stents, and receiving beta-blockers, diuretics, and statin therapy among the three groups. In addition, all of the participants had stable angina with a good thrombolysis in myocardial infarction (TIMI) flow grade. As a result, there were no significant deteriorations or differences between the TIMI flow grade before and after the coronary angiography or angioplasty. Moreover, because all of our electrocardiographic parameters were collected before the examination, we believe that changes in the TIMI flow grade would not affect our results and conclusions.

Multivariate logistic regression analysis was then used to evaluate the effects of plasma FABP4 level and other risk factors for arrhythmia in the patients with an abnormal QTc interval. The results showed positive associations between an abnormal QTc interval and male sex and higher plasma FABP4 level (Table 2).

To determine the effects of FABP4 plasma level on the electrocardiographic parameters, we classified the patients into three groups according to the tertile of FABP4 plasma level as follows: first tertile $\leq 7.4 \mathrm{ng} / \mathrm{mL}$, second tertile 7.5 to $16.25 \mathrm{ng} / \mathrm{mL}$, and third tertile $>16.25 \mathrm{ng} / \mathrm{mL}$. Significant trends were noted in the associations among FABP4 level and heart rate, left ventricular ejection fraction (LVEF), hs-CRP, QTc interval, QRS duration, hypertension, diabetes, HF, and CKD ( $P$ for trend $<0.05$; Table 3 ). In the patients who did not have a MACE, the mean follow-up period was 32.6 months (maximum, 106 months). Nine (6.8\%) of the patients in the highest FABP4 plasma tertile died of any cause, compared to one $(0.8 \%)$ in the lowest tertile. Significant trends were noted among the associations between FABP4 level and MACEs, HF, 
Table 1 Baseline characteristics of the study population stratified by category of QTc prolongation at baseline ${ }^{a}$

\begin{tabular}{|c|c|c|c|c|c|}
\hline Variable & All & Normal & Borderline & Abnormal & $P$-value \\
\hline No & 397 & 171 & 88 & 138 & \\
\hline Sex (male/female) & $308 / 89$ & $122 / 49$ & $74 / 14$ & $112 / 26$ & 0.030 \\
\hline Age (years) & $66.7 \pm 11.5$ & $64.9 \pm 11.8$ & $66.1 \pm 11.4$ & $69.3 \pm 11.0$ & 0.003 \\
\hline Age range & $37-99$ & $37-99$ & 39-93 & $40-94$ & \\
\hline $\mathrm{BMI}\left(\mathrm{kg} / \mathrm{m}^{2}\right)$ & $25.9 \pm 3.7$ & $26.0 \pm 3.6$ & $26.1 \pm 3.5$ & $25.6 \pm 3.9$ & 0.510 \\
\hline Waist (cm) & $91.4 \pm 9.7$ & $91.4 \pm 10.0$ & $93.0 \pm 9.0$ & $90.3 \pm 9.6$ & 0.228 \\
\hline Hypertension (n, \%) & $285(71.8)$ & $106(62.0)$ & $64(72.7)$ & $115(83.3)$ & 0.0002 \\
\hline Hyperlipidemia (n, \%) & $259(65.2)$ & $120(70.2)$ & $60(68.2)$ & $79(57.3)$ & 0.048 \\
\hline Diabetes mellitus ( $n, \%)$ & $166(41.8)$ & $55(32.2)$ & $43(48.9)$ & $68(49.3)$ & 0.003 \\
\hline Heart failure $(n, \%)$ & $65(16.4)$ & $11(6.4)$ & $13(14.8)$ & $41(29.7)$ & $<0.0001$ \\
\hline Current smoking (n, \%) & $188(47.4)$ & $75(43.9)$ & $43(48.9)$ & $70(50.7)$ & 0.461 \\
\hline QTc interval (ms) & $446 \pm 37$ & $416 \pm 19$ & $444 \pm 9$ & $485 \pm 27$ & $<0.0001$ \\
\hline Systolic blood pressure (mmHg) & $133 \pm 22$ & $130 \pm 19$ & $133 \pm 20$ & $135 \pm 26$ & 0.190 \\
\hline Diastolic blood pressure (mmHg) & $77 \pm 14$ & $77 \pm 13$ & $79 \pm 14$ & $77 \pm 14$ & 0.513 \\
\hline Sodium (mEq/L) & $138.6 \pm 4.0$ & $139.1 \pm 3.2$ & $138.8 \pm 3.6$ & $137.9 \pm 4.8$ & 0.018 \\
\hline Potassium (mEq/L) & $3.9 \pm 0.7$ & $3.9 \pm 0.5$ & $3.8 \pm 0.6$ & $4.0 \pm 0.9$ & 0.180 \\
\hline Calcium (mg/dl) & $8.8 \pm 0.9$ & $8.9 \pm 0.8$ & $8.7 \pm 0.7$ & $8.7 \pm 1.1$ & 0.485 \\
\hline Fasting glucose (mg/dl) & $146.8 \pm 81.0$ & $132.0 \pm 60.2$ & $145.1 \pm 66.6$ & $160.3 \pm 81.7$ & 0.002 \\
\hline $\mathrm{HbA1c}(\%)$ & $7.0 \pm 1.8$ & $6.8 \pm 1.5$ & $7.1 \pm 1.5$ & $7.3 \pm 2.1$ & 0.034 \\
\hline T-cholesterol (mg/dl) & $179.7 \pm 43.0$ & $181.3 \pm 39.4$ & $180.6 \pm 41.6$ & $177.2 \pm 48.1$ & 0.701 \\
\hline Triglyceride (mg/dl) & 121.0(89.0-172.0) & 120.5(87.0-172.0) & 122.5(89.0-182.8) & 120.5(92.0-169.0) & 0.764 \\
\hline HDL-cholesterol (mg/dl) & $39.9 \pm 13.4$ & $41.3 \pm 14.5$ & $40.4 \pm 14.8$ & $37.9 \pm 10.7$ & 0.092 \\
\hline LDL-cholesterol (mg/dl) & $104.7 \pm 35.6$ & $107.1 \pm 34.2$ & $104.1 \pm 36.3$ & $102.2 \pm 36.7$ & 0.482 \\
\hline Uric acid (mg/dl) & $6.9 \pm 2.0$ & $6.5 \pm 1.7$ & $6.8 \pm 2.0$ & $7.3 \pm 2.3$ & 0.016 \\
\hline Blood urea nitrogen (mg/dl) & $25.0 \pm 18.8$ & $21.5 \pm 16.7$ & $24.9 \pm 18.6$ & $29.0 \pm 20.5$ & 0.006 \\
\hline Creatinine (mg/dl) & $1.2(1.1-1.6)$ & $1.2(1.1-1.4)$ & $1.2(1.1-1.4)$ & $1.3(1.1-2.5)$ & $<0.0001$ \\
\hline Albumin $(\mathrm{g} / \mathrm{dl})$ & $3.9 \pm 0.4$ & $4.0 \pm 0.4$ & $3.9 \pm 0.4$ & $3.8 \pm 0.4$ & $<0.0001$ \\
\hline Hematocrit (\%) & $39.2 \pm 6.6$ & $40.6 \pm 6.4$ & $39.2 \pm 6.2$ & $37.6 \pm 6.6$ & 0.0003 \\
\hline Hemoglobin $(\mathrm{g} / \mathrm{dl})$ & $13.1 \pm 2.2$ & $13.5 \pm 2.1$ & $13.2 \pm 2.2$ & $12.6 \pm 2.3$ & 0.002 \\
\hline Estimated GFR $\left(\mathrm{ml} / \mathrm{min} / 1.73 \mathrm{~m}^{2}\right)$ & $56.3 \pm 24.0$ & $62.3 \pm 20.3$ & $57.6 \pm 24.0$ & $47.8 \pm 26.1$ & $<0.0001$ \\
\hline Fatty acid-binding protein $4(\mathrm{ng} / \mathrm{mL})$ & $10.6(6.0-22.1)$ & $8.5(5.2-14.4)$ & $10.2(5.3-19.7)$ & $15.9(6.7-41.4)$ & $<0.0001$ \\
\hline Hs-CRP (mg/L) & $2.2(0.7-6.4)$ & $1.5(0.6-4.0)$ & $2.3(0.6-7.2)$ & $4.5(1.2-11.7)$ & $<0.0001$ \\
\hline White blood cell count $\left(\times 10^{9} / \mathrm{L}\right)$ & $8.468 \pm 3.513$ & $7.851 \pm 2.951$ & $8.481 \pm 3.770$ & $9.228 \pm 3.847$ & 0.003 \\
\hline No. of diseased coronary arteries & $1.7 \pm 1.1$ & $1.6 \pm 1.1$ & $1.7 \pm 1.1$ & $1.8 \pm 1.1$ & 0.188 \\
\hline Gensini score & $36.0(15.5-80.5)$ & $41.5(20.0-85.5)$ & $34.0(12.0-64.0)$ & $30.3(15.0-88.4)$ & 0.182 \\
\hline Percutaneous coronary intervention & $323(81.4)$ & $133(77.8)$ & $73(83.0)$ & $117(84.8)$ & 0.264 \\
\hline Number of stent & $0(0-1)$ & $0(0-1)$ & $0(0-1)$ & $0(0-0.3)$ & 0.495 \\
\hline Anti-arrhythmic medication (n, \%) & $113(28.5)$ & $43(25.2)$ & 35 (39.8) & $35(25.4)$ & 0.029 \\
\hline Beta-blockers (n, \%) & $68(17.1)$ & $26(15.2)$ & $22(25.0)$ & $20(14.5)$ & 0.084 \\
\hline Diuretics (n, \%) & $28(7.1)$ & $8(4.7)$ & $7(8.0)$ & $13(9.4)$ & 0.252 \\
\hline Statins (n, \%) & $107(27.0)$ & $47(27.5)$ & $27(30.7)$ & $33(23.9)$ & 0.524 \\
\hline
\end{tabular}

Data are expressed as mean $\pm S D$, number (percentage), or median (interquartile range). HDL high-density lipoprotein, LDL low-density lipoprotein, $H s$ - $C R P$ highsensitivity C-reactive protein. ${ }^{a}$ Classification of QTc prolongation: normal men, $\leq 430 \mathrm{~ms}$; women $\leq 450 \mathrm{~ms}$; borderline men $431-450 \mathrm{~ms}$; women $451-470 \mathrm{~ms}$; abnormal men $\geq 451 \mathrm{~ms}$; women $\geq 471 \mathrm{~ms}$ 
Table 2 Multiple logistic regression analysis with the presence of abnormal QTc interval as the dependent variable

\begin{tabular}{llll}
\hline & $\exp (\mathrm{B})$ & $95 \%$ Confidence Interval & $P$-value \\
\hline Age & 1.02 & $0.99-1.05$ & 0.238 \\
Male sex & 2.70 & $1.16-6.28$ & 0.022 \\
Body mass index & 1.00 & $0.92-1.09$ & 0.973 \\
Systolic blood pressure & 1.00 & $0.99-1.02$ & 0.686 \\
Fasting glucose & 1.00 & $0.99-1.01$ & 0.133 \\
Total cholesterol & 1.00 & $0.99-1.00$ & 0.209 \\
Estimated glomerular filtration rate & 0.99 & $0.98-1.01$ & 0.671 \\
Sodium & 0.97 & $0.90-1.04$ & 0.373 \\
Potassium & 0.83 & $0.50-1.36$ & 0.454 \\
Calcium & 0.84 & $0.58-1.22$ & 0.362 \\
Use of anti-arrhythmic drugs & 0.63 & $0.24-1.64$ & 0.341 \\
Use of antihypertensive drugs & 0.72 & $0.22-2.43$ & 0.599 \\
Fatty acid-binding protein 4 & 1.02 & $1.00-1.03$ & 0.017 \\
\hline
\end{tabular}

and mortality ( $P$ for trend $<0.05$; Table 3 ). However, no significant differences were found in coronary intervention results with quantitative angiographic analysis including minimal lumen diameter, final minimal lumen diameter, acute gain, percent stenosis, and reference lumen diameter among the three groups of plasma FABP4 (all $P>0.05$ ).

A positive association was observed between FABP4 level and QTc interval (beta $=0.267, P<0.0001$; Fig. 1). Pearson's correlation analysis revealed that FABP4 plasma level was positively correlated with age, SBP, DBP, levels of creatinine, fasting glucose, hs-CRP, HbA1c, and blood urea nitrogen, and QTc interval. In addition, eGFR, and levels of HDL-C, hemoglobin, and hematocrit were negatively correlated with levels of FABP4 (Table 4).

\section{Discussion}

In the current study, we found that plasma FABP4 levels were independently associated with abnormal QTc interval in patients with stable angina and CKD. The associations between plasma FABP4 and QTc prolongation still persisted after controlling for conventional risk factors including age, sex, SBP, BMI, use of anti-arrhythmic and antihypertensive medications, eGFR, and levels of potassium, sodium, calcium, fasting glucose, and total cholesterol. Furthermore, we also found that the level of hs-CRP was higher in the patients with QTc prolongation, and that hs-CRP was well correlated with FABP4 levels. These findings are in agreement with current evidence regarding the association between ventricular arrhythmias and inflammation $[37,38]$.

The secretion of FABP4 from visceral or subcutaneous adipose tissue, epicardial fat tissue, or macrophages can affect heart dysfunction through paracrine and endocrine pathways. The expression of FABP4 has been shown to be strongly induced during adipocyte differentiation [39], and thus several studies have proposed that this molecule can be used as a marker of adipocyte differentiation [40, 41]. Several studies have shown the induction of FABP4 expression when monocytes differentiate to macrophages in a manner similar to adipocytes, and a wide range of proinflammatory stimuli have been shown to regulate its expression in these cells $[42,43]$. In addition, FABP4 has been reported to induce the formation of foam cells, increase the accumulation of cholesterol ester, and induce inflammatory responses in macrophages through activation of the JNK-AP-1 and IKK-NF- $\mathrm{KB}$ pathways $[44,45]$. Our findings suggest that the inflammatory activity reflected by hs-CRP level was a strong etiologic factor for the higher levels of plasma FABP4 in the patients with QTc prolongation. FABP4 has recently been shown to be associated with an increased cardiometabolic risk, and previous studies have reported both clinical and experimental evidence that FABP4 is a relevant factor in atherosclerosis and CAD [24, 46]. Furthermore, it has been directly associated with cardiac alterations such as left ventricular hypertrophy and both systolic and diastolic cardiac dysfunction [47]. Despite the strong evidence showing the effect of plasma FABP4 concentration on cardiovascular diseases, the relationship between plasma FABP4 level and electrocardiographic parameters are unknown, especially its arrhythmogenic effect on the QTc interval.

Patients with CKD have been reported to have a high frequency of prolonged QTc interval [48]. Moreover, a prolonged QTc interval has been shown to be a marker of defective cardiac repolarization, and an important cause of sudden cardiac death and cardiac arrhythmias. The major factors contributing to repolarization defects include the administration of drugs, genetic defects, HF, 
Table 3 Prevalence of major adverse cardiovascular events and all-cause mortality and electrocardiographic parameters according to fatty acid-binding protein 4 level

\begin{tabular}{|c|c|c|c|c|}
\hline Parameter & First tertile & Second tertile & Third tertile & $P$ for trend \\
\hline FABP4 (ng/mL) & $\leq 7.4$ & $7.5-16.25$ & $>16.25$ & \\
\hline Number & 132 & 133 & 132 & \\
\hline LVEF (\%) & $62.7 \pm 11.5$ & $62.9 \pm 11.1$ & $57.9 \pm 12.8$ & 0.001 \\
\hline $\mathrm{Hs}-\mathrm{CRP}(\mathrm{mg} / \mathrm{L})$ & $1.3(0.4-4.6)$ & $1.8(0.7-4.0)$ & $4.4(1.5-10.1)$ & 0.043 \\
\hline White blood cell count $\left(\times 10^{9} / \mathrm{L}\right)$ & $8.212 \pm 2.991$ & $8.011 \pm 3.322$ & $9.188 \pm 4.058$ & 0.356 \\
\hline \multicolumn{5}{|l|}{ ECG parameters } \\
\hline Heart Rate (bpm) & $72.0 \pm 16.8$ & $74.1 \pm 16.2$ & $82.4 \pm 16.6$ & 0.001 \\
\hline PR interval (ms) & $168.2 \pm 34.0$ & $166.4 \pm 33.6$ & $172.7 \pm 32.4$ & 0.812 \\
\hline QRS duration (ms) & $95.1 \pm 13.4$ & $96.0 \pm 18.9$ & $99.5 \pm 19.9$ & 0.020 \\
\hline QT interval (ms) & $403.1 \pm 39.5$ & $402.1 \pm 38.2$ & $399.9 \pm 45.0$ & 0.743 \\
\hline QTc interval (ms) & $436.0 \pm 30.7$ & $440.2 \pm 34.4$ & $461.5 \pm 39.2$ & $<0.0001$ \\
\hline \multicolumn{5}{|l|}{ Medication (n, \%) } \\
\hline Anti-arrhythmic medication & $34(25.8)$ & $38(28.6)$ & $41(31.1)$ & 0.634 \\
\hline Beta-blockers & $22(16.7)$ & $23(17.3)$ & $23(17.4)$ & 0.985 \\
\hline Diuretics & $12(9.1)$ & $6(4.5)$ & $10(7.6)$ & 0.333 \\
\hline Statins & $38(28.8)$ & $36(27.1)$ & $33(25.0)$ & 0.786 \\
\hline Hypertension (n, \%) & 77 (58.3) & $99(74.4)$ & $109(82.6)$ & $<0.0001$ \\
\hline Dyslipidemia (n, \%) & $84(63.6)$ & $92(69.2)$ & $83(62.9)$ & 0.501 \\
\hline Diabetes (n, \%) & $31(23.5)$ & $53(39.9)$ & $82(62.1)$ & $<0.0001$ \\
\hline Heart failure (n, \%) & $14(10.6)$ & $15(11.3)$ & $36(27.3)$ & 0.0002 \\
\hline Chronic kidney disease $^{\mathrm{a}}(\mathrm{n}, \%)$ & $36(27.3)$ & $58(43.6)$ & $105(79.6)$ & $<0.0001$ \\
\hline MACEs $(n, \%)$ & $41(31.1)$ & $47(35.3)$ & $66(50.0)$ & 0.004 \\
\hline All-cause Mortality (n, \%) & $1(0.8)$ & $4(3.0)$ & $9(6.8)$ & 0.026 \\
\hline \multicolumn{5}{|l|}{ Non-fatal outcome (n, \%) } \\
\hline Heart failure & $6(4.6)$ & $6(4.5)$ & $15(11.4)$ & 0.028 \\
\hline Target lesion revascularization & $28(21.2)$ & $32(24.1)$ & $31(23.5)$ & 0.562 \\
\hline Recurrent myocardial Infarction & $6(4.6)$ & $5(3.8)$ & $11(8.3)$ & 0.180 \\
\hline
\end{tabular}

Data are expressed as mean $\pm S D$, number (percentage), or median (interquartile range). FABP4 fatty acid-binding protein 4, LVEF left ventricular ejection fraction, $H s$-CRP high-sensitivity C-reactive protein, ECG electrocardiography, QTC corrected QT, MACEs major adverse cardiovascular events. ${ }^{a}$ Chronic kidney disease was defined as an eGFR< $60 \mathrm{~mL} / \mathrm{min} / 1.73 \mathrm{~m}^{2}$

diabetes mellitus, sex, renal failure, hypokalemia, and hypothyroidism. Repolarization defects have also been reported in the sudden death of athletes. However, the precise pathophysiological mechanisms behind a prolonged QT interval have yet to be elucidated. In particular, as renal function deteriorates, levels of proinflammatory cytokines increase [49]. Patients with CKD have been reported to have higher chronic inflammation, which could be another potential mechanism resulting in cardial fibrosis, vascular damage, sympathetic overactivity, and ion channel malfunction, which can increase the risk of cardiac arrhythmias and death $[14,50]$. In addition, higher levels of markers of inflammation have been associated with the outcomes of patients with acute coronary syndromes and the risk of atherosclerotic complications [51]. Furthermore, previous studies have shown associations between CRP and cytokines such as IL- 6 and atrial fibrillation and other arrhythmias in the absence of CAD [13], raising the possibility of a direct electrophysiological effect. In support of this hypothesis, the modulation of ion channel function and production of arrhythmias by cytokines such as platelet-activating factor have been described [12]. Consistent with these findings, our findings suggest that inflammatory activity as reflected by hs-CRP level was a strong etiologic factor for the higher levels of plasma FABP4 in the patients with QTc prolongation. In addition, the patients with higher levels of plasma FABP4 had a lower LVEF, higher prevalence of CKD, and higher hs-CRP level compared with the other groups. FABP4 has been associated with adiposity and metabolic disorders, and it has been reported to be a novel predictor of cardiovascular 


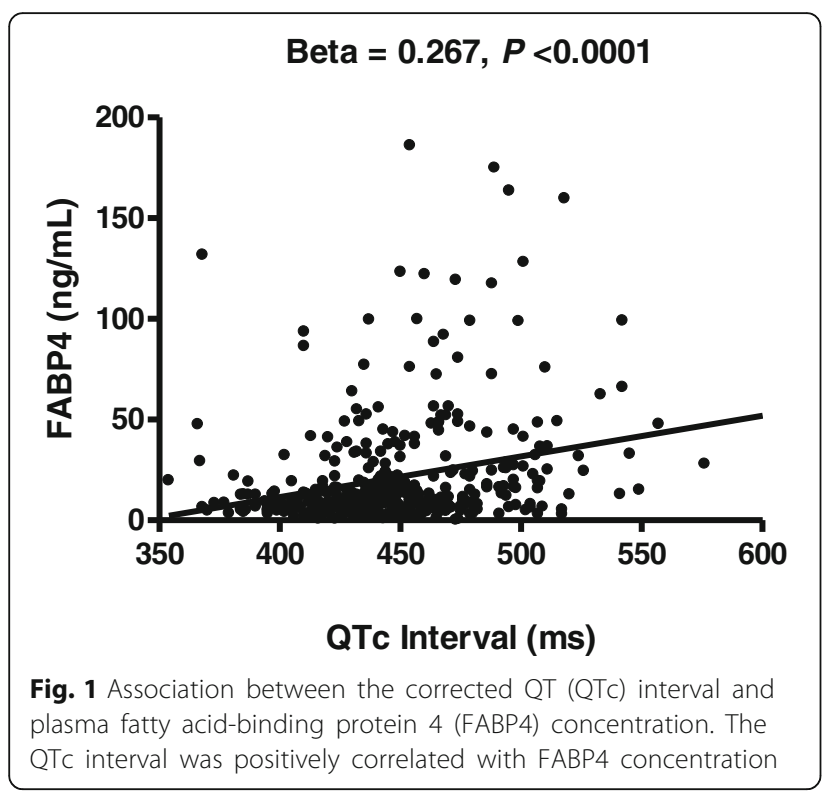

mortality in end-stage renal disease [52]. In addition, previous studies using animal models have indicated that FABP4 plays a significant role in several aspects of the metabolic syndrome, including insulin resistance, type 2 diabetes, and atherosclerosis, through its action at the

Table 4 Pearson's correlation analysis of fatty acid-binding protein 4with clinical laboratory data

\begin{tabular}{lll}
\hline Variable & $r$ & $P$-value \\
\hline Age & 0.113 & 0.025 \\
Body mass index & 0.043 & 0.391 \\
Systolic blood pressure & 0.201 & 0.0001 \\
Diastolic blood pressure & 0.124 & 0.014 \\
QTc interval & 0.267 & $<0.0001$ \\
Fasting glucose & 0.169 & 0.001 \\
HbA1c & 0.136 & 0.013 \\
Total-cholesterol & -0.015 & 0.767 \\
Triglycerides & 0.080 & 0.111 \\
HDL-cholesterol & -0.112 & 0.028 \\
LDL-cholesterol & -0.094 & 0.062 \\
Uric acid & 0.106 & 0.084 \\
Blood urea nitrogen & 0.381 & $<0.0001$ \\
Creatinine & 0.589 & $<0.0001$ \\
eGFR & -0.594 & $<0.0001$ \\
Hemoglobin & -0.407 & $<0.0001$ \\
Hematocrit & -0.400 & $<0.0001$ \\
Hs-CRP & 0.280 & $<0.0001$ \\
White blood cell count & 0.073 & 0.145 \\
Current smoking & 0.102 & 0.098 \\
\hline HDL high-densty lipopten & &
\end{tabular}

$H D L$ high-density lipoprotein, $L D L$ low-density lipoprotein, eGFR estimated glomerular filtration rate, $\mathrm{Hs}$-CRP high-sensitivity C-reactive protein interface of metabolic and inflammatory pathways in adipocytes and macrophages [46, 53-55]. The results of the present study support the idea [44-46] that FABP4 may act through an inflammation response to play an important role in the pathophysiology of QTc interval prolongation in patients with stable angina and CKD. Furthermore, in the present study, the patients with higher levels of plasma FABP4 also had higher rates of hypertension, diabetes, and HF, thereby raising the possibility that FABP4 directly contributes to cardiac repolarization defects and is associated with QTc prolongation.

We also found that there were significant trends in the associations among FABP4 level and MACEs and allcause mortality. Moreover, we found that plasma FABP4 levels were most strongly positively correlated with blood urea nitrogen and creatinine. This suggests that the level of plasma FABP4 increases with the progression of renal failure. Furuhashi et al. suggested that the concentration of plasma FABP4 may be a marker of metabolic syndrome, and that it could also be used in patients with end-stage renal disease and as a novel predictor of cardiovascular mortality in patients at high risk of atherosclerotic cardiovascular events [52].

The current study has some limitations. First, as a cross-sectional study, we could not make a cause-andeffect conclusion between increased plasma FABP4 levels and QTc prolongation. Further long-term follow-up studies are needed to clarify the role of plasma FABP4 in QTc prolongation. Second, in our study, all the patients' ECGs parameters including QT interval and QTc were measured by same medical technician using identical computer-based method, as a result, we did not evaluate the variability of measured QT interval. Third, in accordance with the guidelines of the Bureau of National Health Insurance, serum magnesium is not routinely measured in patients with stable angina, and thus magnesium data were not available in this study. Previous studies have reported that low serum magnesium has been linked to increased risk of atrial and ventricular arrhythmias and cardiovascular mortality [56, 57]. However, no association has been reported between serum magnesium levels and QTc interval [33]. Fourth, if the study population had different diseases (e.g. acute coronary syndrome and myocardial infarction), the diverse disease severity and condition of the study population may have impacted the results. To avoid selection bias, we chose individuals with stable angina for this study, thus the results of the present study might not be generalizable to other populations. Furthermore, in the present study, we found that an abnormal QTc interval was positively associated with male sex. Due to the small number of female patients we could not perform subgroup analysis by sex, and further studies are 
required to clarify this issue. Moreover, whether FABP4 is associated with the expression of cardiomyocyte electrophysiology, cardiac ion channels, and the underlying mechanisms are still unclear. Further future studies are warranted to elucidate these issues.

\section{Conclusion}

The results of this study indicated that a prolonged QTc interval in patients with stable angina and CKD was correlated with an elevated FABP4 level. Further studies are required to investigate the associations between FABP4 level and QTc interval.

\begin{abstract}
Abbreviations
ANOVA: Analysis of variance; BMI: Body mass index; CAD: Coronary artery disease; CKD: Chronic kidney disease; CRP: C-reactive protein; DBP: Diastolic blood pressure; ECGs: Electrocardiograms; eGFR: estimated glomerular filtration rate; FABP4: Fatty acid binding protein 4; HDL-C: High density lipoprotein- cholesterol; HF: Heart failure; hs: high-sensitivity; IQR: Interquartile range; LDL-C: Low density lipoprotein- cholesterol; LVEF: Left ventricular ejection fraction; MACEs: Major adverse cardiac events; MLD: Minimal lumen diameter; QCA: Quantitative coronary angiography; QTc: Corrected QT; SBP: Systolic blood pressure; TGs: Triglycerides
\end{abstract}

\section{Acknowledgements}

The authors are grateful to the staff and members of the heart care teams for their assistance with various measurements and other organizational aspects of this study.

\section{Role of funder in this study}

The funder in this study is E-Da Hospital, and the role was only to financially support this research with no role in the design of this study and no role during its execution, analyses, interpretation of the data, or decision to submit the results.

\section{Authors' contributions}

Of the 11 authors, CPW is the first author who conceived and designed the study and interpreted the data. CCH, YJL, WHT, ITT and JYH contributed to drafting of the manuscript or revising it critically for important intellectual content. WCH, CCW and THY acquired data, drafted the manuscript and designed the study. Dr. FMC participated in the design of the study and performed the statistical analysis. Dr. YCL is the corresponding author and contributed to the final approval of the manuscript as submitted. All authors read and approved the final version of the manuscript.

\section{Funding}

This study was supported by E-Da Hospital of the Republic of China, Taiwan (grant no. EDAHP106050).

\section{Availability of data and materials}

Due to the regulations of our Institutional Research Ethics Committees and Institutional Review Boards, the detailed data of the study subjects cannot be disclosed publicly. However, the data set can be provided on request by any journal review board after approval from the Institutional Research Ethics Committees.

\section{Ethics approval and consent to participate}

The Ethics Committee of I-Shou University E-Da Hospital approved this study (EDAH IRB No. EMRP-105-051). All patients gave written consent to participate in this study.

\section{Consent for publication}

Not applicable.

\section{Competing interests}

The authors declare that they have no competing interests.

\section{Author details}

${ }^{1}$ Division of Cardiology, I-Shou University, Kaohsiung 82445, Taiwan. ${ }^{2}$ Division of Gastroenterology and Hepatology, I-Shou University, Kaohsiung 82445, Taiwan. ${ }^{3}$ Division of Endocrinology and Metabolism, Department of Internal Medicine, E-Da Hospital, I-Shou University, No. 1, Yi-Da Rd, Jiau-Shu Village, Yan-Chao District, Kaohsiung 82445, Taiwan. ${ }^{4}$ Department of Emergency, E-Da Hospital, I-Shou University, Kaohsiung 82445, Taiwan. ${ }^{5}$ Division of Cardiology, Department of Internal Medicine, National Yang-Ming University Hospital, Yilan 26058, Taiwan. ${ }^{6}$ School of Medicine for International Students, College of Medicine, I-Shou University, Kaohsiung 82445, Taiwan.

${ }^{7}$ Department of Nutrition, Institute of Biotechnology and Chemical Engineering, College of Medicine, I-Shou University, Kaohsiung 82445, Taiwan. ${ }^{8}$ Lee's Endocrinologic Clinic, Pingtung 90000, Taiwan.

Received: 27 September 2018 Accepted: 12 June 2019

Published online: 24 June 2019

\section{References}

1. Fox CS, Evans JC, Larson MG, Kannel WB, Levy D. Temporal trends in coronary heart disease mortality and sudden cardiac death from 1950 to 1999: the framingham heart study. Circulation. 2004;110:522-7.

2. de Bruyne MC, Hoes AW, Kors JA, Hofman A, van Bemmel JH, Grobbee DE. QTC dispersion predicts cardiac mortality in the elderly: the Rotterdam Study. Circulation. 1998:97:467-72.

3. Schwartz PJ, Wolf S. QT interval prolongation as predictor of sudden death in patients with myocardial infarction. Circulation. 1978;57:1074-7.

4. Dekker JM, Crow RS, Hannan PJ, Schouten EG, Folsom AR, ARIC Study. Heart rate-corrected QT interval prolongation predicts risk of coronary heart disease in black and white middle-aged men and women: The ARIC study. Am Coll Cardiol. 2004;43:565-71.

5. Robbins J, Nelson JC, Rautaharju PM, Gottdiener JS. The association between the length of the QT interval and mortality in the cardiovascular health study. Am J Med. 2003:115:689-94.

6. Im SW, Lee MK, Lee HJ, Oh SI, Kim HL, Sung J, et al. Analysis of genetic and non-genetic factors that affect the QTc interval in a Mongolian population: the GENDISCAN study. Exp Mol Med. 2009;41:841-8.

7. Busjahn A, Knoblauch H, Faulhaber HD, Boeckel T, Rosenthal M, Uhlmann R et al. QT interval is linked to 2 long-QT syndrome loci in normal subjects. Circulation. 1999:99:3161-4.

8. Nie Y, Zou J, Liang Y, Shen B, Liu Z, Cao X, et al. Electrocardiographic abnormalities and QTC interval in patients undergoing hemodialysis. PLoS One. 2016;11:e0155445.

9. Tbahriti HF, Meknassi D, Moussaoui R, Messaoudi A, Zemour L, Kaddous A, et al. Inflammatory status in chronic renal failure: the role of homocysteinemia and pro-inflammatory cytokines. World J Nephrol. 2013:2:31-7.

10. Jara A, Mezzano S. Vascular damage in chronic kidney disease. Rev Med Chil. 2008;136:1476-84

11. Hoffman BF, Feinmark SJ, Guo SD. Electrophysiologic effects of interactions between activated canine neutrophils and cardiac myocytes. J Cardiovasc Electrophysiol. 1997;8:679-87.

12. Hoffman BF, Guo SD, Feinmark SJ. Arrhythmias caused by platelet activating factor. J Cardiovasc Electrophysiol. 1996;7:120-33.

13. Chung MK, Martin DO, Sprecher D, Wazni O, Kanderian A, Carnes CA et al. C-reactive protein elevation in patients with atrial arrhythmias: inflammatory mechanisms and persistence of atrial fibrillation. Circulation. 2001;104:2886-91

14. Parekh RS, Plantinga LC, Kao WH, Meoni LA, Jaar BG, Fink NE, et al. The association of sudden cardiac death with inflammation and other traditional risk factors. Kidney Int. 2008;74:1335-42.

15. Makowski L, Hotamisligil GS. Fatty acid binding proteins-the evolutionary crossroads of inflammatory and metabolic responses. J Nutr. 2004;134: 2464S-8S.

16. Stejskal D, Karpisek M. Adipocyte fatty acid binding protein in a Caucasian population: a new marker of metabolic syndrome? Eur J Clin Investig. 2006; 36:621-5

17. Bagheri R, Qasim AN, Mehta NN, Terembula K, Kapoor S, Braunstein S, et al. Relation of plasma fatty acid binding proteins 4 and 5 with the metabolic syndrome, inflammation and coronary calcium in patients with type-2 diabetes mellitus. Am J Cardiol. 2010;106:1118-23. 
18. Xu A, Wang Y, Xu JY, Stejskal D, Tam S, Zhang J, et al. Adipocyte fatty acidbinding protein is a plasma biomarker closely associated with obesity and metabolic syndrome. Clin Chem. 2006;52:405-13.

19. Xu A, Tso AW, Cheung BM, Wang Y, Wat NM, Fong $\mathrm{CH}_{\text {, et al. }}$ Circulating adipocyte-fatty acid binding protein levels predict the development of the metabolic syndrome: a 5-year prospective study. Circulation. 2007;115:1537-43

20. Lamounier-Zepter V, Look C, Alvarez J, Christ T, Ravens U, Schunck WH, et al. Adipocyte fatty acid-binding protein suppresses cardiomyocyte contraction: a new link between obesity and heart disease. Circ Res. 2009; 105:326-34.

21. Tso AW, Lam TK, Xu A, Yiu KH, Tse HF, Li LS, et al. Serum adipocyte fatty acid-binding protein associated with ischemic stroke and early death. Neurology. 2011;76:1968-75.

22. Tso AW, Xu A, Sham PC, Wat NM, Wang Y, Fong CH, et al. Serum adipocyte fatty acid binding protein as a new biomarker predicting the development of type 2 diabetes: a 10-year prospective study in a Chinese cohort. Diabetes Care. 2007;30:2667-72.

23. Miyoshi T, Onoue G, Hirohata A, Hirohata S, Usui S, Hina K, et al. Serum adipocyte fatty acid- binding protein is independently associated with coronary atherosclerotic burden measured by intravascular ultrasound. Atherosclerosis. 2010:211:164-9.

24. Jin J, Peng DQ, Yuan SG, Zhao SP, Ning XH, Wang SH, et al. Serum adipocyte fatty acid binding proteins and adiponectin in patients with coronary artery disease: the significance of A-FABP/ adiponectin ratio. Clin Chim Acta. 2010; 411:1761-5.

25. Djoussé L, Bartz TM, IX JH, Kochar J, Kizer JR, Gottdiener JS, et al. Fatty acidbinding protein 4 and incident heart failure: the cardiovascular health Study. Eur J Heart Fail. 2013;15:394-9.

26. Cabré A, Lázaro I, Girona J, Manzanares JM, Marimón F, Plana N, et al. Plasma fatty acid-binding protein 4 increases with renal dysfunction in type 2 diabetic patients without microalbuminuria. Clin Chem. 2008:54:181-7.

27. Kong X, Ma Y, Chen J, Luo Q, Yu X, Li Y, et al. Chinese eGFR investigation collaboration. Evaluation of the chronic kidney disease epidemiology collaboration equation for estimating glomerular filtration rate in the Chinese population. Nephrol Dial Transplant. 2013;28:641-51.

28. American Diabetes Association. Diagnosis and classification of diabetes mellitus. Diabetes Care. 2012;35(Suppl 1):S64-71.

29. Expert Panel on Detection, Evaluation, and Treatment of High Blood Cholesterol in Adults. Executive summary of the third report of the National Cholesterol Education Program (NCEP) expert panel on detection evaluation, and treatment of high blood cholesterol in adults (adult treatment panel III). JAMA. 2001;285:2486-97.

30. Gensini GG. Amore meaningful scoring system for determining the severity of coronary heart disease. Am J Cardiol. 1983;51:606.

31. Wang CP, Hsu HL, Hung WC, Yu TH, Chen YH, Chiu CA, et al. Increased epicardial adipose tissue (EAT) volume in type 2 diabetes mellitus and association with metabolic syndrome and severity of coronary atherosclerosis. Clin Endocrinol. 2009;70:876-82.

32. Rautaharju PM, Surawicz B, Gettes LS, Bailey JJ, Childers R, Deal BJ, et al. AHA/ACCF/HRS recommendations for the standardization and interpretation of the electrocardiogram: part IV: the ST segment, T and $U$ waves, and the QT interval: a scientific statement from the American Heart Association Electrocardiography and Arrhythmias Committee, Council on Clinical Cardiology; the American College of Cardiology Foundation; and the Heart Rhythm Society endorsed by the International Society for Computerized Electrocardiology. J Am Coll Cardiol. 2009:53:982-91.

33. Naksuk N, Hu T, Krittanawong C, Thongprayoon C, Sharma S, Park JY, et al. Association of Serum Magnesium on mortality in patients admitted to the intensive cardiac care Unit. Am J Med. 2017:130:229. e5-229.e13.

34. Salvi V, Karnad DR, Panicker GK, Natekar M, Hingorani P, Kerkar V, et al. Comparison of 5 methods of QT interval measurements on electrocardiograms from a thorough QT/QTC study: effect on assay sensitivity and categorical outliers. J Electrocardiol. 2011;44:96-104.

35. Malik M, Hnatkova K, Batchvarov V, Gang Y, Smetana P, Camm AJ. Sample size, power calculations, and their implications for the cost of thorough studies of drug induced QT interval prolongation. Pacing Clin Electrophysiol. 2004;27:1659-69.
36. Straus SM, Kors JA, De Bruin ML, van der Hooft CS, Hofman A, Heeringa J, et al. Prolonged QTc interval and risk of sudden cardiac death in a population of older adults. J Am Coll Cardiol. 2006;47: 362-7.

37. Sano K, Watanabe E, Hayano J, Mieno Y, Sobue Y, Yamamoto M, et al. Central sleep apnoea and inflammation are independently associated with arrhythmia in patients with heart failure. Eur J Heart Fail. 2013;15:1003-10.

38. Vasilets LM, Tuev AV, Agafonov AV, Ratanova EA, Krivaia AA, Trenogina $\mathrm{KV}$, et al. Ventricular arrhythmias and arterial hypertension: role of inflammatory biomarkers in arrhythmia development. Vestn Ross Akad Med Nauk. 2012;10:12-7.

39. Bernlohr DA, Bolanowski MA, Kelly TJ Jr, Lane MD. Evidence for an increase in transcription of specific mRNAs during differentiation of 3T3-L1 preadipocytes. J Biol Chem. 1985;260:5563-7.

40. Bernlohr DA, Doering TL, Kelly TJ Jr, Lane MD. Tissue specific expression of p422 protein, a putative lipid carrier, in mouse adipocytes. Biochem Biophys Res Commun. 1985;132:850-5.

41. Yang WW, Christy RJ, Cook JS, Kelly TJ, Lane MD. Mechanism of regulation of the 422(aP2) gene by cAMP during preadipocyte differentiation. Proc Natl Acad Sci U S A. 1989;86:3629-33.

42. Wang XQ, Yang K, He YS, Lu L, Shen WF. Receptor mediated elevation in FABP4 levels by advanced glycation end products induces cholesterol and triacylglycerol accumulation in THP-1 macrophages. Lipids. 2011:46:479-86.

43. Kazemi MR, McDonald CM, Shigenaga JK, Grunfeld C, Feingold KR. Adipocyte fatty acid-binding protein expression and lipid accumulation are increased during activation of murine macrophages by toll-like receptor agonists. Arterioscler Thromb Vasc Biol. 2005;25:1220-4.

44. Makowski L, Brittingham KC, Reynolds JM, Suttles J, Hotamisligil GS. The fatty acid-binding protein, aP2, coordinates macrophage cholesterol trafficking and inflammatory activity. Macrophage expression of aP2 impacts peroxisome proliferator-activated receptor gamma and IkappaB kinase activities. J Biol Chem. 2005;280:12888-95.

45. Hui X, Li H, Zhou Z, Lam KS, Xiao Y, Wu D, et al. Adipocyte fatty acidbinding protein modulates inflammatory responses in macrophages through a positive feedback loop involving c-Jun $\mathrm{NH2}$-terminal kinases and activator protein-1. J Biol Chem. 2010;285:10273-80.

46. Makowski L, Boord JB, Maeda K, Babaev VR, Uysal KT, Morgan MA, et al. Lack of macrophage fatty-acidbinding protein aP2 protects mice deficient in apolipoprotein E against atherosclerosis. Nat Med. 2001;7:699-705.

47. Rodríguez-Calvo R, Girona J, Alegret JM, Bosquet A, Ibarretxe D, Masana L. Role of the fatty acid-binding protein 4 in heart failure and cardiovascular disease. J Endocrinol. 2017:233:R173-R84.

48. Bignotto LH, Kallás ME, Djouki RJ, Sassaki MM, Voss GO, Soto CL, et al. Electrocardiographic findings in chronic hemodialysis patients. J Bras Nefrol. 2012;34:235-42.

49. Vianna HR, Soares CM, Tavares MS, Teixeira MM, Silva AC. Inflammation in chronic kidney disease: the role of cytokines. J Bras Nefrol. 2011;33:351-64.

50. Shamseddin MK, Parfrey PS. Mechanisms of the cardiorenal syndromes. Nat Rev Nephrol. 2009:5:641-9.

51. Libby P, Ridker PM, Maseri A. Inflammation and atherosclerosis. Circulation. 2002;105:1135-43

52. Furuhashi M, Ishimura S, Ota H, Hayashi M, Nishitani T, Tanaka M, et al. Serum fatty acid-binding protein 4 is a predictor of cardiovascular events in end-stage renal disease. PLoS One. 2011;6:e27356.

53. Furuhashi M, Fucho R, Görgün CZ, Tuncman G, Cao H, Hotamisligil GS Adipocyte/ macrophage fatty acid-binding proteins contribute to metabolic deterioration through actions in both macrophages and adipocytes in mice. J Clin Invest. 2008:118:2640-50.

54. Maeda K, Cao H, Kono K, Gorgun CZ, Furuhashi M, Uysal KT, et al. Adipocyte/ macrophage fatty acid binding proteins control integrated metabolic responses in obesity and diabetes. Cell Metab. 2005;1:107-19.

55. Boord JB, Maeda K, Makowski L, Babaev VR, Fazio S, Linton MF, et al. Combined adipocyte-macrophage fatty acid-binding protein deficiency improves metabolism, atherosclerosis, and survival in apolipoprotein Edeficient mice Circulation. 2004:110:1492-8.

56. Del Gobbo LC, Imamura F, Wu JH, de Oliveira Otto MC, Chiuve SE, Mozaffarian D. Circulating and dietary magnesium and risk of cardiovascular disease: a systematic review and meta-analysis of prospective studies. Am J Clin Nutr. 2013;98:160-73. 
57. Adamopoulos C, Pitt B, Sui X, Love TE, Zannad F, Ahmed A. Low serum magnesium and cardiovascular mortality in chronic heart failure: a propensity-matched study. Int J Cardiol. 2009;136:270-7.

\section{Publisher's Note}

Springer Nature remains neutral with regard to jurisdictional claims in published maps and institutional affiliations.

Ready to submit your research? Choose BMC and benefit from:

- fast, convenient online submission

- thorough peer review by experienced researchers in your field

- rapid publication on acceptance

- support for research data, including large and complex data types

- gold Open Access which fosters wider collaboration and increased citations

- maximum visibility for your research: over $100 \mathrm{M}$ website views per year

At BMC, research is always in progress. 